\title{
Client-Server Model
}

\author{
Haroon Shakirat Oluwatosin \\ School of Computing Universiti Utara Malaysia Kedah, Malaysia
}

\begin{abstract}
Client- server is a system that performs both the functions of client and server so as to promote the sharing of information between them. It allows many users to have access to the same database at the same time, and the database will store much information. This paper will provide information about client-server model in terms of its introduction, architecture, recent development and issues.
\end{abstract}

Keywords: client-server, 2-tier, 3-tier, Interprocess communication, thick client, middleware SaaS, PaaS, IaaS.

\section{Introduction}

With the advancement in technology, Web is becoming very much more important in our daily lives, in which virtually everything we do nowadays involves the use of web. More so, the application of Web is not limited to computers but it is opened to different kinds of intelligent digital devices, for example the mobile ones. Also, the architecture of the Web is the Client-Server model, in which as a result the communication between the client and server is the first thing we should be concerned about [1]. Client/server system has increasingly minimized application development time by dividing functions of sharing information into both the client and server. The client is the requester while the server is the provider of service. In most client-server environment, the data processing is handled by the server, and the results are returned to the clients, which is made to speed up the rate of performance [2].For example, in a workstation, a printer can be attached to a computer (representing the clients) while other computers sharing from it are the server.

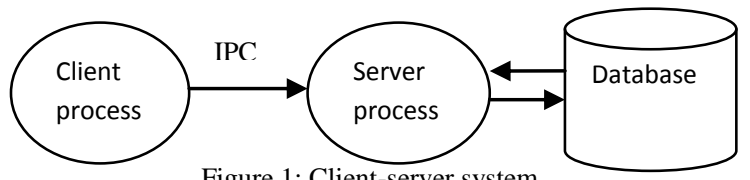

Figure 1: Client-server system

\section{What Is Client-Server System}

In the computing world today, client-server system has become so popular because it is being used virtually every day for different applications. Some of the standardized protocols that client and servers use to communicate with themselves include: File Transfer Protocol (FTP), Simple Mail Transfer Protocol (SMTP) and Hypertext Transfer Protocol (HTTP). Thus, Client-server system can be define as a software architecture made up of both the client and server, whereby the clients always send requests while the server responds to the requests sent[3]. Client-server provides an inter-process communication because it involves the exchange of data from both the client and server whereby each of them performs different functions $[3,8]$.

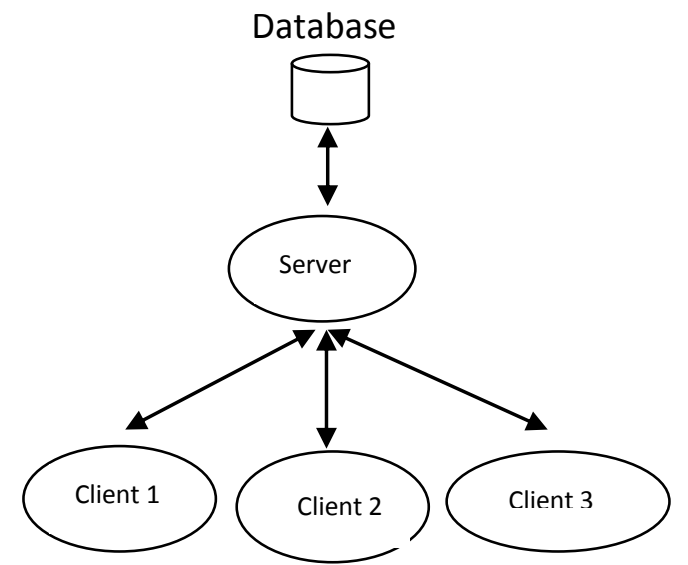

Figure 2: Interprocess communication among client and server

\section{A. Benefits of client server model}

- It splits the processing of application across multiple machines [2]. 
- It allows easier sharing of resources from client to servers.

- It reduces data replication by storing data on each server instead of client.

\section{B. Example of applications using client-server system}

File transfer: This is the transmission of files between the client and server. It also allows storing of files on the server. Files such as movies, images, music can be stored.

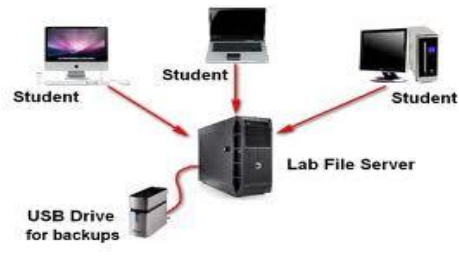

Figure 3: File server

-Mail transfer: This is the transfer of messages such as the email using the Mail Transfer Protocol (MTP).

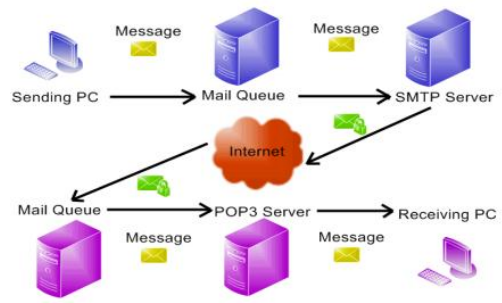

Figure 4: Mail transfer

- $\quad$ Hypertext Transfer Protocol (HTTP): Is the transferring of multimedia files such as images, text between the client and server [3]. HTTP is use to improve communication between the client and server, by serving as a request-response protocol.

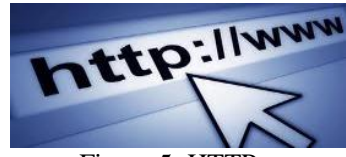

Figure 5: HTTP

\section{Client-Server Systems Architecture}

Client-server architecture is usually made up of the; application server, database server and PC. The two main architectures are the 2-tier and 3-tier architecture.

- 2-tier client-server system architecture: This is an architecture which involves only the Database server and a client PC. In 2-tier architecture, the users will run applications on their PC (Client), which connects through a network to the server. The client application runs both the coding and business logic, and then displays output to the user. It is also called thick client.

-It is considered when the client has access to the database directly without involving any intermediary.

-It is also used to perform application logic whereby the application code will be assigned to each of the client in the workstation.

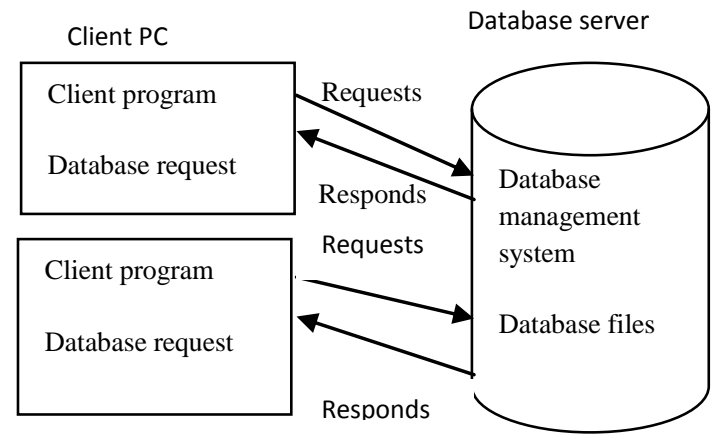

Figure 6: 2-tier client-server architecture. 
- 3-tier client-server system architecture: This architecture involves the client PC, Database server and Application server. 3-tier architecture can be extended to N-tier whereby it involves more application servers.

In this architecture, the client contains presentation logic only, whereby less resources and less coding are needed by the client.

It supports one server being in charge of many clients and provides more resources in the server [2]. It involves an intermediary (Application server) also known as middleware.

Middleware: The 3-tier architecture involves an application server which serves as a middleware between the client PC and database server. The middleware tier is separate software running on a separate machine and performs application logic [9].
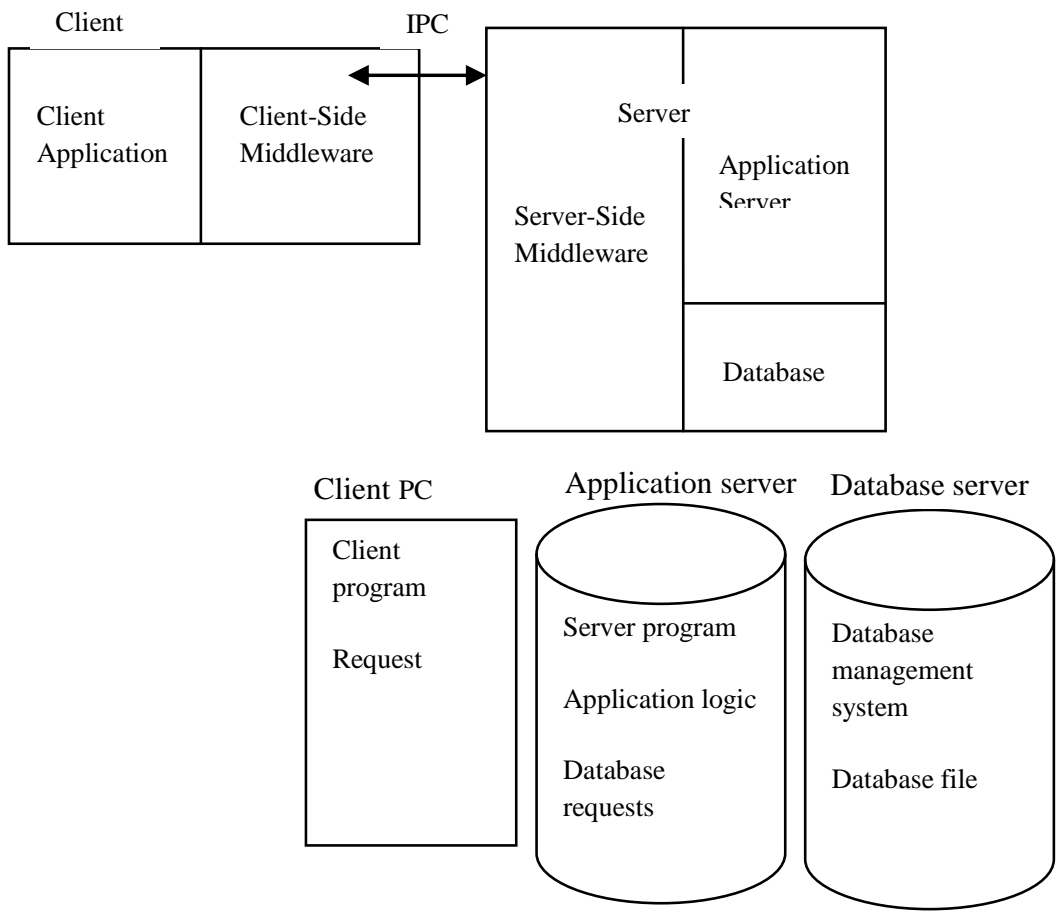

Figure 8: 3-tier client-server architecture

\section{Recent Development}

Client server continues to gain more mainstream adoption as more companies move into the cloud. Market growth in different types of service such as distributed and cloud computing [7].

- Cloud computing: There are many definitions of cloud computing, but one of the most common ones describe cloud computing as a group of distributed computer that provides services and resources through the internet [10]. There are three main services that can be offered by cloud, which are:

- Infrastructure as Service (IaaS): The products offered by this mode are through the Internet, such as servers.

- Platform as a Service (PaaS): In the platform of cloud computing, the services are provided through the Internet by cloud providers. Unlike, the traditional method in which each application requires the use of hardware, software, operating system etc.

- Software as a Service (SaaS): in this layer, you don't need to install or maintain software; the applications are delivered through the Internet [11].

The general importance of Client Server system in Cloud computing is horizontal scalability to millions of virtual machines, examples of applications that uses this technology include; Google Apps (Gmail, Google talk) [7,10]. For example, in a university setting which has a computer centre that caters for the student, lecturers, software developer and researchers by providing them with the required software, hardware and development tools. The university decide to shift to the use of cloud, such that the students and lecturers can use the service of SaaS and IaaS clouds provider and then the software used by them will be saved on the servers of SaaS cloud and can be accessed online, then any other hardware or additional requirement will be executed online by the IaaS cloud provider. The software developer can finally use all the software and hardware they need for the development and hosting through PaaS cloud provider. 


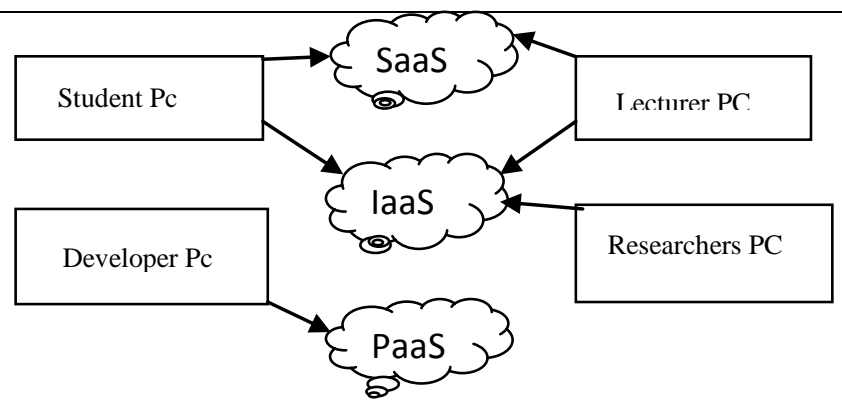

Figure 9: The structure of users in a university using the cloud computing

- Mobile agent: This is an entity working with the computer, it has the ability to reason, can run in another remote site with the support of network, gather results, search results, work with other sites and finally return to its own site after the completion of its assigned tasks [12]. They are called mobile agents because they have the ability to move from one computer to another computer through the network. Mobile agents serve as a direct extension of the client server approach. In the client-server approach, each communication entities have a specific role to play like the server offering services and the client using them. Mobile agents is a form of advancement to client-server system and it has more benefit, some of which are [13]:

- Communication: In client-server system, the servers do not have the ability to communicate with each other, whereas mobile agent works like a peer to peer entity and can either act as a client or server.

- Persistence: When the mobile agent is created, it has the ability to work on its own so it is not affected when other nodes fails.

- Efficiency: This will reduce the traffic caused in client-server system during the process of sending messages, because the mobile agent has the ability to pre-process data locally and choose the important information to send.

- Fault tolerance: In the case of client-server system, when a server is down the connection is lost. But the mobile agents have the ability to continue working in the node if network fails.

\section{Issues And Challenges In Client-Server System}

There are lots of issues in a client-server system, some of which include:

The number of people: In most client-server network, few servers are always involved which make the setup looks like a waste of time. A client server network is quite difficult to set up, so it requires lots of servers so as not to render the application useless.

It requires skilled expertise: A lot of client-server network are not well build and managed. Setting up a client server network is so complex, so it requires skilled technician and maintenance engineers to handle it.

The servers are quite expensive: The servers are being designed to meet high standard for it to be reliable and have better performance. Getting the good design and architecture for the client-server system, contributes to it being expensive.

- $\quad$ Security: The most of all is the security issue [4]. The client operating system is easily accessed by servers, and this expose the client system to a number of problems [5]. The message being exchange between the client and server leads to lot of security challenges. A lot of things are encompassing by the security challenges, some of which include: physical damage, threats and virus attack. In other to have a secured network, there are some basic rules to be followed such as;

The Audit network

The discretionary control

The object re-use

Authorization and identification [6].

\section{Conclusion}

The Automated Teller Machine (ATM) is a typical example of Client Server system, imagine a world without it, transfer and withdrawal of cash will be tedious and also the workload on the staffs of the bank will not be able to meet the demand of their customers, this will lead to poor performance, reduced output and relatively low profit from the banks income.

In conclusion, the client-server system encompasses different areas both in networking and database management due to its structural functionalities, it is essential to know that the advent of the technology is a breakthrough in the IT world and also in the business world because without the application of this technology, work process would be difficult to execute and might take longer time. 


\section{Reference}

[1]. Zhang, H. (2013). Architecture of Network and Client-Server model. arXiv preprint arXiv:1307.6665.

[2]. Kambalyal, C. (2010). 3-tier architecture. Retrieved On, 2.

[3]. Kratky, S., \& Reichenberger, C. (2013). Client/Server Development based on the Apple Event Object Model. Atlanta.

[4]. Dewire, D. T. (1993). Client/server computing. McGraw-Hill, Singapore

[5]. Jenkins, N. (1996). Client/server Unleashed. Sams, USA

[6]. Davis, P.T.(Editor) (1996). Securing Client/Server Computer Networks. McGraw-Hill, USA

[7]. Cloud Computing - Issues, Research and Implementations" by Mladen A. Vouk, Journal of Computing and Information Technology - CIT 16, (2008), 4, 235-246 doi:10.2498/cit.1001391

[8]. Kratky, S., \& Reichenberger, C. (2013). Client/Server Development based on the Apple Event Object Model. Atlanta.

[9]. Fong, J., \& Hui, R. (1999). Application of middleware in the three tier client/server database design methodology. Journal of the Brazilian Computer Society, 6(1), 50-64.

[10]. Grossman, R. (2009). The case for cloud computing. IT Professional, 11(2), 23-27

[11]. Sultan, N. (2010). Cloud computing for education: A new dawn?. International Journal of Information Management, 30(2), 109116.

[12]. Outtagarts, A. (2009). Mobile agent-based applications: A survey. IJCSNS International Journal of Computer Science and Network Security, 9(11), 331-339.

[13] P. E. Renaud, Introduction To Client/Server Systems: A Practical Guide For Systems Professionals, Wiley \& Sons, 1993. 